\title{
Analyse d'article
}

\section{Roger MIEUSSET}

Centre de Stérilité Masculine, Hôpital Paule de Viguier

Toulouse

Transient scrotal hyperthermia and levonorgestrel enhance testosterone-induced spermatogenesis suppression in men through increased germ cell apoptosis.

The Journal of Clinical Endocrinology, 2007, 92 : 3292-3304.

Wang C., Cui Y-G., Wang X-H. et al.

L'équipe de C. Wang et R.S. Swerdloff avait précédemment démontré chez le rat et le singe que la suppression de la spermatogenèse par le testostérone ( $T$ ) était amplifiée par une exposition transitoire du scrotum à une hyperthermie (Lue et al., 2000, 2006). Les auteurs ont aussi montré que cette augmentation de suppression était due à une accélération de l'apoptose passant par la voie de signalisation mitochondriale. L'apoptose induite par le traitement associant testostérone et chaleur survient principalement au niveau des spermatides rondes et des spermatocytes pachytènes mais à des stades différents, avec une atteinte des stades précoces (I-IV) et tardifs (XI et XII) pour la chaleur, alors que la testostérone affecte les stades hormono-sensibles (VII et VIII) chez le rat. L'association du traitement par la chaleur et la testostérone affecte tous les stades de la spermatogenèse, aboutissant à une suppression plus marquée de la production de spermatozoïdes. Cette spécificité de l'atteinte des stades est moins nette chez le singe, bien que les spermatides rondes et les spermatocytes pachytènes soient les cellules germinales les plus sensibles à l'apoptose due à la voie mitochondriale.

Par ailleurs, les progestatifs augmentent la suppression des gonadotrophines et de la spermatogenèse obtenue par les androgènes seuls dans les essais de contraception masculine, ce résultat provenant essentiellement des effets additifs des deux stéroïdes sur la suppression des gonadotrophines.
Le mécanisme de la suppression de la spermatogenèse par les hormones n'est toutefois pas clairement élucidé, des études précédentes de cette équipe ayant montré une apoptose accrue des cellules germinales par la T exogène, alors que d'autres auteurs utilisant l'association $T+$ progestatif ont décrit une réduction du nombre de spermatogonies $B$ et un défaut de spermiation amenant une rétention des spermatozoïdes chez les rongeurs, le singe et l'homme. C'est pourquoi l'équipe de Wang et Swerdloff pose la question suivante : l'ajout d'un second 'choc' tel qu'un agent physique (la chaleur) ou un autre agent suppresseur des gonadotrophines (un progestatif) au traitement par T exogène peut-il entraîner une accélération de l'apoptose résultant en une suppression accrue de la spermatogenèse ?

\section{MATERIEL ET METHODES}

Patients : 72 hommes volontaires, chinois $(35,6 \pm 5,4$ ans) inclus dans l'étude après un bilan (clinique, hématologique, lipidique) normal et 3 examens de sperme normaux à 2 semaines d'intervalle (OMS).

Protocole : randomisation des volontaires en 4 groupes (n=18/groupe) : Groupe 1 : undécanoate de testostérone (UT) seul ; Groupe 2 : chaleur seule; Groupe 3 : UT + chaleur ; Groupe 4 : UT + lévonorgestrel (LNG).

Durée de l'étude : phase de traitement $=18$ semaines (sem.) ; phase de suivi post traitement $=12 \mathrm{sem}$. ; durée totale de l'étude $=30 \mathrm{sem}$.

Modalités du traitement hormonal : Groupes 1, 3 et 4 : au 1 er jour du traitement, 1 injection IM de 1000mg UT suivie par 1 injection IM de $500 \mathrm{mg}$ UT aux semaines 6 et 12 ; injections faites par le personnel de l'équipe réalisant l'étude. Le choix d'un intervalle de 6 semaines pour les injections de $500 \mathrm{mg}$ UT (une injection toutes les $4 \mathrm{sem}$. induisant une concentration $<1 \times 10^{6} \mathrm{spz} / \mathrm{ml}$ ) est d'obtenir une suppression partielle de la spermatogenèse pour évaluer les effets additifs potentiels de la chaleur ou des progestatifs au traitement par testostérone seule. 
Les hommes du Groupe 4 prennent en plus chaque matin 1 comprimé de 250 ?g de LNG pendant toute la durée de la phase de traitement.

Modalités du traitement par la chaleur : fait sous contrôle de l'équipe réalisant l'étude. Immersion du scrotum dans un bain d'eau dont la température est progressivement amenée puis maintenue à $43^{\circ} \mathrm{C}$ (contrôle simultané des températures du scrotum et de l'eau). La température de $43^{\circ} \mathrm{C}$ atteinte, l'homme reste assis dans le bain pendant $30 \mathrm{~min} /$ jour sur 6 jours consécutifs. Ce protocole de chauffage scrotal repose sur les études menées précédemment chez l'homme par Watanabe (1959) et par l'équipe de Rock et Robinson (1965; 1967).

Les hommes des Groupes 2 et 3 sont soumis quotidiennement à un tel chauffage des jours 8 à 13, soit durant toute la sem. 2 de la phase de traitement.

\section{SUIVI}

Clinique : bilan de départ puis aux sem. $6,12,18$ et 30 .

Sperme : analyse toutes les 3 sem. pendant la phase de traitement puis 4 sem. pendant la phase de suivi post traitement, prolongée si nécessaire jusqu'à la récupération d'une concentration de spermatozoïdes (spz) identique aux valeurs de départ ou $>20 \times 10^{6} \mathrm{spz} / \mathrm{ml}$.

Hormones : FSH, LH, T, T libre, SHBG, et Inhibine B mesurées à $\mathrm{J} 0, \mathrm{~J} 8, \mathrm{~J} 14$ puis toutes les $3 \mathrm{sem}$. pendant la phase post traitement.

Qualité de vie : un questionnaire validé est rempli par tous les hommes chaque jour pendant 7 jours successifs avant le début de l'étude et aux sem. $6,12,18$ et $30 ; 4$ domaines sont renseignés : désir sexuel, plaisir sexuel, activité sexuelle, humeur.

Biopsie testiculaire : réalisée chez 4 volontaires avant le traitement, puis chez 4 volontaires de chacun des 4 groupes aux sem. 2 et 9 de la phase de traitement. La sem. 2 a été choisie pour refléter les modifications morphologiques du testicule et le taux d'apoptose à la fin du traitement par la chaleur. La sem. 9 devait représenter les effets de la suppression des gonadotrophines et de la T sur le testicule.

Une seule biopsie a été faite par homme portant sur un seul testicule. Tout homme ayant constitué sa famille s'est vu proposer une biopsie lors de l'inclusion. Un refus de la biopsie ne constituait pas un critère d'exclusion.

La biopsie était effectuée sous anesthésie locale, à ciel ouvert, avec un prélèvement de tissu (jusqu'à $100 \mathrm{mg}$ ) partagé en 3 part : une fixée au Bouin (étude de l'apoptose), une congelée dans l'azote liquide (analyse des protéines), la dernière conservée en milieu spécifique (étude de l'expression des gènes).

Approbation du protocole : obtenue auprès des instances respectives des 2 équipes impliquées dans l'étude (Nanjing, Chine ; Los Angeles, Californie). Compensation financière allouée à chaque homme pour perte de temps de travail et frais de transport selon recommandations et standards locaux, approuvée par les institutions respectives.
Paramètres évalués : apoptose des cellules germinales, morphologie testiculaire.

\section{RESULTATS}

\section{Concentration de spermatozoïdes}

Sem. 3 : la concentration de spz diminue dans tous les groupes sauf pour UT seul.

Chaleur : la diminution de la concentration de $\mathrm{spz}$ est maximale à la sem. 6 puis revient progressivement aux valeurs de départ à la sem. 12. Le traitement par UT seul supprime la spermatogenèse plus lentement pour atteindre un effet maximal à la sem. 18 .

L'association UT + chaleur supprime de façon marquée la spermatogenèse à la sem. 6 , niveau maintenu jusqu'à la sem. 18.

Aux sem. 3 et 6 , la chaleur seule et UT + chaleur ont un effet suppresseur sur la spermatogenèse significativement plus marqué sur la concentration de spz que UT seul.

Ala sem. 9, la suppression de la spermatogenèse est accrue pour UT seul et UT + chaleur alors que le groupe chaleur seule est déjà en phase de récupération. A la sem. 9, la réduction de la concentration de spz est significativement moindre pour UT seul et pour chaleur seule que pour UT + chaleur.

LNG : aux sem. 3 et 6, UT + LNG induit une réduction de la concentration de spz significativement plus grande que UT seul, mais significativement moins grande que UT + chaleur. L'effet UT + LNG est maintenu jusqu'à la sem. 18.

Récupération : la concentration moyenne de spz est revenue aux valeurs de départ à la sem. 30 pour chacun des groupes.

\section{Mobilité et morphologie des spz}

Le pourcentage de spz mobiles et le pourcentage de spz de forme normale suivent le même profil que la concentration de spz. Ces deux paramètres reviennent pratiquement à la normale à la sem. 30 .

\section{Proportion d'hommes avec oligospermie sévère $\left(<3 \times 10^{6} \mathrm{spz} / \mathrm{ml}\right)$ et azoospermie}

Chaleur seule : aucune concentration de $\mathrm{spz}<3 \times 10^{6} \mathrm{spz} / \mathrm{ml}$ au cours du traitement.

Azoospermie atteinte à la sem. 18 pour $7 / 18$ hommes avec UT seul, 6/18 avec UT + chaleur et $17 / 18$ avec UT + LNG.

Oligospermie atteinte significativement pour plus d'hommes avec UT + chaleur que dans les autres groupes à la sem. 9.

\section{Hormones}

Les moyennes de $T$ et $T$ libre ne sont significativement modifiées dans aucun des groupes. La SHBG moyenne est non modifiée avec chaleur, mais significativement légèrement diminuée avec UT et UT + chaleur, et significativement plus réduite ( $45 \%$ des valeurs de base) avec UT + LNG que dans les autres groupes avec UT. La moyenne de l'inhibine B n'est pas modifiée avec chaleur ou UT seuls mais significativement diminuée avec UT + chaleur (15\%) et UT + LNG (34\%). 


\section{Innocuité}

Aucun effet secondaire avec chaleur. Après biopsie testiculaire, 3 hommes ont présenté des douleurs au point d'incision, et un quatrième un hématome léger sans nécessité de traitement. Acné modérée chez 4 hommes à la fin du traitement sans acné avant le traitement.

Légère mais significative augmentation de l'hémoglobine avec chaleur, UT + LNG, et UT + chaleur, et de l'hématocrite avec UT, chaleur, et UT + chaleur. Diminution faible mais significative du volume testiculaire moyen à la sem. 18 avec UT $(4 \%)$, UT + chaleur $(3 \%)$, et UT + LNG $(2,5 \%)$, mais pas avec chaleur.

\section{Qualité de vie}

Absence de modification de l'humeur pour tous les groupes. Mais les scores moyens pour le désir sexuel, le plaisir sexuel, l'activité sexuelle, la fréquence et la qualité satisfaisante des érections sont tous significativement augmentés au cours de l'étude, sans différence entre les groupes à une seule exception : le groupe UT + chaleur n'a montré aucune modification du plaisir sexuel et diffère significativement du groupe chaleur pour lequel les scores moyens sont les plus augmentés.

\section{Morphologie testiculaire}

Pas de différence majeure après 2 sem. de traitement, sauf dans le groupe UT + chaleur : réduction significative du diamètre des tubes séminifères (TS). Cependant, après 9 sem. de traitement, différences plus marquées dans les groupes UT + chaleur et UT + LNG : UT : réduction du diamètre des TS, et diminution plus marquée du nombre de spermatocytes et de spermatides tubes séminifères. Mêmes modifications avec UT + LNG mais avec en plus une réduction supplémentaire du nombre de cellules germinales.

L'altération maximale de la spermatogenèse survient avec UT + chaleur : la réduction du diamètre des TS est plus importante, les altérations morphologiques plus étendues avec oblitération complète de la lumière des TS, épaississement de la membrane basale, et absence totale de spermatides. Confirmation de ces résultats à l'analyse quantitative.

Morphologie identique à celle des témoins pour chaleur à 9 sem.

\section{Activation de l'apoptose}

Evolution quantitative de l'apoptose faite sur les biopsies testiculaires après 2 sem. de traitement ; incidence de l'apoptose des cellules germinales exprimée en nombre pour 100 cellules de Sertoli.

Sem. 2 : aucune modification avec UT ou UT + LNG. Par contre, chaleur et UT + chaleur entraînent une augmentation significative de l'apoptose par rapport au pré-raitement.

\section{DISCUSSION}

Dans leur protocole, les auteurs ont choisi d'augmenter l'intervalle entre les injections UT de façon à pouvoir étudier les effets additifs potentiels d'un agent physique (chaleur) ou hormonal (progestatif) sur la suppression de la spermatogenèse induite par UT seule. Avec ce protocole, ils retrouvent dans leur étude des résultats cohérents avec les traitements hormonaux (UT et UT + LNG) pour les modifications hormonales et la suppression de la spermatogenèse, avec une azoospermie ou une oligospermie sévère chez seulement $50 \%$ des hommes.

Pour ce qui est du traitement par la chaleur, les auteurs ont utilisé la technique de chauffage des testicules publiée par Watanabe en 1959 (bain de siège de 30 min dans une eau à $43^{\circ} \mathrm{C}$ ) pendant 6 jours consécutifs dans leur étude, ces 6 jours constituants la sem. 2 de la phase de traitement. Ils observent une réduction de la production de spz commençant à la sem. 3 , maximale à la sem. 6 , avec un retour aux valeurs de départ à la sem. 12.

Les auteurs montrent que l'association de UT pendant 18 sem. plus chaleur pendant la sem. 2 du traitement par UT augmente la suppression de la spermatogenèse aux sem. 3 à 9 par rapport à UT seul. Ils confirment aussi que l'ajout d'un progestatif (LNG) à UT augmente l'effet obtenu par UT seul sur la décroissance de la production de $s p z$, cet effet n'étant pas associé à des taux sériques plus élevés de testostérone totale ou libre mais à une suppression plus efficace et plus complète des taux de gonadotrophines avec UT + LNG. Enfin, la diminution significative des taux d'inhibine $B$ avec UT + chaleur et UT + LNG, par comparaison à UT seul ou chaleur seule, suggère une dysfonction plus sévère des cellules de Sertoli.

Les auteurs démontrent ainsi (et c'était l'objectif de leur travail) que l'association de 2 'chocs', la testostérone exogène ('choc 1 ') et une hyperthermie testiculaire transitoire ou l'ajout d'un progestatif ('choc 2') ont une effet additif sur la réduction de la production de $\mathrm{spz}$.

L'étude morphologique des biopsies testiculaires permet aux auteurs de montrer d'une part que la diminution de la spermatogenèse observée à la sem. 9 avec UT + chaleur affecte principalement les spermatocytes et les spermatides; mais ils mettent aussi en évidence que l'association UT + chaleur entraîne une diminution rapide et marquée de la concentration de $\mathrm{spz}$ qui est associée à une activation significative de l'apoptose des cellules germinales en 2 sem. de traitement, démontrant ainsi que l'apoptose est un déterminant important de la suppression de la spermatogenèse induite par la testostérone ou la chaleur.

Cependant, d'autres auteurs ont monté que les premières cellules atteintes étaient les spermatogonies $B$ après 2 sem. de traitement par énanthate de $\mathrm{T}$ seul ou par implant de d'acétate de médroxy progestérone (McLachan et al., 2002), ce qui implique que l'inhibition de la prolifération des spermatogonies est aussi un déterminant important de la production de spz après traitement par testostérone et/ou progestatif.

Dans leur étude, les auteurs n'observent pas d'absence de spermiation, quel que soit le type de traitement ; ces résultats sont discordants avec ceux précédemment rapportés chez les rongeurs et les primates, dont l'homme (McLachan et al., 
2002 ; O'Donnel et al., 2001) qui montraient une absence de spermiation caractérisée par la présence de spermatides allongées dans les profondeurs de l'épithélium séminifère, phénomène pouvant jouer un rôle important dans la suppression de la spermatogenèse par contraception hormonale. Les auteurs n'ont pas d'explication à cette discordance, si ce n'est que l'absence de spermiation est un événement 'dynamique', alors que la biopsie testiculaire constitue un point événementiel 'statique'. IIs avancent, toutefois, que la diminution marquée de la concentration de spz à la sem. 3 avec chaleur, UT + chaleur, et UT + LNG suggère qu'un défaut de spermiation est probable.

Les mécanismes pour expliquer la suppression de la spermatogenèse par différents traitements impliquent ainsi l'inhibition de la prolifération des spermatogonies, l'activation de l'apoptose des cellules germinales et l'absence de spermiation.

\section{COMMENTAIRES}

Ce travail apporte des informations nouvelles dans le domaine de la contraception masculine. Tout d'abord, il présente des explications à la suppression de la spermatogenèse par les différents traitements utilisables en contraception masculine comme touchant 3 processus différents : la multiplication de spermatogonies, l'activation de l'apoptose, et la perturbation de la spermiation, dernière étape de la spermatogenèse. Mais ce travail est aussi important dans le fait qu'il est le premier à utiliser une approche expérimentale en contraception masculine associant les hormones et la chaleur.

Si comme le disent les auteurs, les résultats du traitement par la chaleur (hyperthermie transitoire par chauffage scrotale à $43^{\circ} \mathrm{C}$ ) sont bien semblables à ceux rapportés par Watanabe (1959) en termes de dynamique et d'intensité de l'effet sur la production de $s p z$, ils ne peuvent, dans le temps de l'étude, être considérés comme semblables à ceux rapportés avec les techniques de chauffage testiculaire par isolation scrotale (Rock et Robinson, 1965 ; Robinson et Rock, 1967) ou par cryptorchidie artificielle (Mieusset et al., 1985 ; 1991) pour lesquelles les durées d'exposition à la chaleur sont beaucoup plus longues ( $8 \mathrm{sem}$. à 24 mois) et l'intensité du chauffage (valeur atteinte par la température testiculaire) moindre. II s'agit en effet de 2 modèles différents d'élévation de la température testiculaire, le premier $\left(43^{\circ} \mathrm{C}\right)$ créant un état d'hyperthermie supra physiologique, la température testiculaire atteinte étant supérieure à la température physiologique du testicule, les seconds induisant une température testiculaire qui, bien que supérieure à la température testiculaire physiologique $\left(+1\right.$ à $\left.2^{\circ} \mathrm{C}\right)$, reste inférieure à la température corporelle normale $\left(37^{\circ} \mathrm{C}\right)$. Ces 2 modèles induisent des effets différents, concernant entre autres le débit sanguin testiculaire et d'autres processus dont la régulation ou l'expression sont dépendantes de la température testiculaire.

Bien que les auteurs expliquent clairement dans leur article que leur objectif n'est pas d'utiliser l'association hormone + chaleur comme méthode de contraception masculine, on peut tout à fait envisager une telle approche associant testostérone exogène et chaleur. Et cela de deux façons différentes.
D'une part, les auteurs montrent une réduction plus rapide et plus intense de la concentration de spz avec UT + chaleur qu'avec UT seul ou avec UT + LNG. L'association UT + chaleur devrait ainsi permettre une atteinte plus rapide de l'effet contraceptif (azoospermie) que celui obtenu actuellement avec l'utilisation contraceptive de la seule testostérone.

D'autre part, l'association d'un traitement par testostérone et chaleur en pratique contraceptive réelle permettrait d'espacer les doses de testostérone, et donc de limiter les effets indésirables liés à la prise au long cours d'androgènes exogènes. Et cela d'autant plus que, selon les modalités de cette étude, la seule exposition quotidienne du scrotum de 30 min/jour sur 6 jours consécutifs (pendant la sem. 2 du traitement hormonal) montrant un effet maximum de l'association sur la suppression de la spermatogenèse à la sem. 6 , c'est-à-dire 3 sem. après la fin du chauffage, le traitement par la chaleur pourraient être répété toutes les 3 sem., ce qui devrait permettre, par conséquence, d'augmenter la durée de l'intervalle entre les injections de testostérone...

Note. L'ensemble des références utilisées dans cette analyse d'article provient de l'article analysé. 